\title{
Transcriptomics in the NICHE
}

The combination of photoactivatable fluorescent markers with single-cell RNAseq allows transcriptome analysis of cells from specific tissue locations.

The immune system is made up of many cell types that respond to stimuli and changing conditions. Technologies to characterize immune cell types and their activation state at the transcriptional level have evolved rapidly in the last few years. But the immune system is all about location-who is where, when - and these advanced approaches cannot link transcriptome-level information to a cell's niche or location within a tissue. To enable capture of both sets of information with high precision and throughput, NICHEseq combines photoactivatable markers with massively parallel single-cell RNA-seq.

Immune cells alter their behavior based on cues from their surroundings or physical interaction with nearby cells. But approaches to combine a cell's spatial location with its phenotype have been limited by sequencing depth, by requiring prior knowledge of differentially expressed genes, or by the structure of the tissue of interest. In NICHE-seq, cells in specific regions of an organ can be tracked by photoactivating markers using two-photon microscopy. Following tissue dissociation and fluorescently activated cell sorting of labelled cells, their transcriptomes can be accessed by scRNA-seq.

Ido Amit, Ziv Schulman and Matteo Iannacone and colleagues, who developed NICHE-seq, used it to study the B-cell follicles and T-cell zone in lymph nodes, areas known to host rare cell types. They used transgenic mice expressing photoactivatable GFP and photoactivated cells within $\mathrm{B}$ or $\mathrm{T}$ cell areas in lymph nodes. Once isolated and sorted, scRNA-seq of the labelled cells revealed the cell types present, including enrichment of B cells and T cells in their respective regions, as expected. And follow- ing infection with a murine virus, scRNA-seq showed the upregulation of an antiviral program in the cells.

In the spleen, the researchers used a viral strain that drives GFP expression. This allowed them to mark cells in infected niches and characterize differences in cell-type composition and transcriptional programs to those in noninfected niches in the spleen.

Because many immune cells originate in the bone marrow, bone marrow transfers from PA-GFP mice to irradiated mice can also be used with NICHE-seq. Expanding the range of photoactivatable markers would add versatility and resolution, but NICHE-seq is already likely to be of great use to immunologists and tissue anatomists.

\section{Irene Jarchum}

\section{RESEARCH PAPERS}

Medaglia, C. et al. Spatial reconstruction of immune niches by combining photoactivatable reporters and scRNA-seq. Science 358, 1622-1626 (2017). 